\title{
Identification and clinical implications of a novel pathogenic variant in the $G J B 2$ gene causes autosomal recessive non-syndromic hearing loss in a consanguineous Iranian family
}

\author{
Mahbobeh Koohiyan* \\ Cancer Research Center, Shahrekord University of Medical Sciences, Shahrekord, Iran.
}

\begin{abstract}
SUMMARY Mutations in the GJB2 gene, which encodes the connexin26 protein and is involved in inner ear homeostasis, are the most common cause of autosomal recessive non-syndromic hearing loss (ARNSHL) in many populations. This study was aimed to determine the molecular etiology in a consanguineous Iranian family affected by profound ARNSHL. A comprehensive family history was obtained, and clinical evaluations and pedigree analysis were performed in the family with 4 affected members. After extraction of genomic DNA, the entire coding region of GJB2 was directly sequenced in all family members. In silico analyses were also performed using available software tools. Sanger sequencing results showed a novel rare homozygous variant (c.109_110insG) in the GJB2 gene. This frameshift variant in exon 2 of the GJB2 gene fulfills the criteria of being categorized as pathogenic according to the American College of Medical Genetics and Genomics (ACMG) guideline. Confirmation was done with the co-segregation study and checking the frequency of the novel variant in 100 ethnically matched normal control subjects. The present study suggests that investigation of GJB2 mutations may still be useful to determine the etiology of HL in Iran.
\end{abstract}

Keywords autosomal recessive non-syndromic hearing loss, GJB2, ACMG guideline, Iran

\section{Introduction}

Hearing loss (HL) is the most frequent sensory impairment, affecting 1 in 1,000 neonates worldwide (http://hearing.screening.nhs.uk/nationalprog). Many environmental factors, such as drug exposure, bacterial or viral infections and trauma, can cause HL; however, a significant proportion of cases is due to genetic factors. It is estimated that $70 \%$ of HL includes non-syndromic forms (NSHL), where the hearing deficit is the only clinical phenotype (1). Autosomal recessive mode of inheritance (ARNSHL) is highly heterogeneous, for which over 100 mapped loci are known to be involved (http://hereditaryhearingloss.org). Despite this, mutations in one single locus, DFNB1 (13q1112) which contains the GJB2 (NM_004004.5) and GJB6 (NM_001110219.2) genes, account for 50\% of the etiology in many European populations (2). Their identification of the first GJB2 mutations causing hearing loss was soon followed by screenings that revealed a high frequency of GJB2 mutations among subjects with NSHL (3). The gene is 5513 bp long and contains only two exons with exon 1 being untranslated. To date, 361 mutations in the GJB2 gene have been identified with variable frequency among disparate world populations (http://www.hgmd.cf.ac.uk). c.35delG is the most common mutation in many populations such as Iran $(4,5)$. Other mutations have a more specific origin. In Japanese, $235 \mathrm{delC}$ is more prevalent ( 6 ) and c.167delT is common in Ashkenazi Jews (7). Genetic diagnosis of ARNSHL is complicated, in terms of cost-effectiveness, due to the extreme genetic heterogeneity of this condition (8-11). So the high rate of GJB2 related HL in most populations $(12,13)$, coupled with the simple structure of the gene, which facilitated the design of different molecular tests, made screening for GJB2 mutations the first step of genetic testing of ARNSHL (14). Here, we report a novel frameshift variant in a consanguineous Iranian family affected by profound ARNSHL. Our finding would be a great supplement for the GJB2 pathogenic mutations and would make genetic counseling available for this family.

\section{Methods}

\subsection{Participants and molecular genetic testing}

Clinical examination was carried out on the four affected subjects; family CHM-1 from a village 
located in southwest Iran. A comprehensive family history, including age of onset, exposure to ototoxic drugs during pregnancy and childhood infections, was obtained. Further clinical examinations such as temporal bone CT scan, thyroid ultrasound scan and thyroid hormone assays were also carried out to rule out the involvement of other body organs and syndromic HL forms (Figure 1). Air and bone conduction pure tone audiometry from 250 to $8000 \mathrm{~Hz}$ was obtained from all affected. All members of this family were sampled for further molecular studies. Blood samples were collected after written informed consent was obtained from all individuals or their legal guardians (if patients were under 18). Written parental consent to publish was also obtained. The study was approved by the review boards and ethics committees of the Shahrekord University of Medical Sciences.

Genomic DNA of subjects was extracted from peripheral blood lymphocytes using the standard salting out procedure (15). Purity and concentration of DNA samples were determined with a $1.2 \%$ agarose gel and spectrophotometer (Nanolytik, Dusseldorf, Germany). GJB2 was screened for the coding region mutations

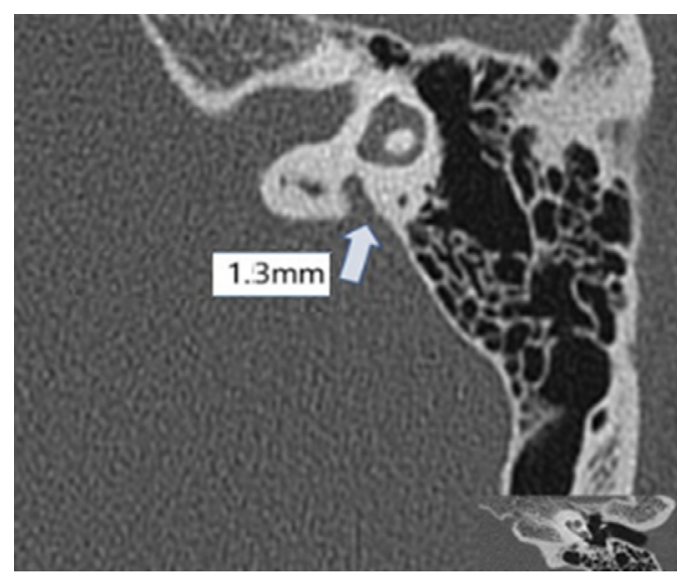

Figure 1. Temporal bone CT image of IV: 1 . based on the criteria of diameter greater than $1.5 \mathrm{~mm}$ between the outer vestibular aqueduct and the vestibule of the total port foot or the midpoint of the isthmus is abnormal. (exon 2) using direct sequencing. The following primers were used for amplification of exon 2 of the GJB2 gene: F1 (5'-GCTTACCCAGACTCAGAGAAG-3') and R1 (5'-CTACAGGGGTTTCAAATGGTTGC-3'). The PCR reaction was performed as follows: $8 \mu \mathrm{L}$ of Master Mix 2x (containing, $\mathrm{MgCl}_{2}$, Taq DNA polymerase, and d NTPs (Applied Biosystems), $0.45 \mu \mathrm{L}$ of each of the primers (10 pM), $2 \mu \mathrm{L}$ DNA (50 ng), adjusted to $18 \mu \mathrm{L}$ using $\mathrm{ddH}_{2} \mathrm{O}$. The amplification was initiated by denaturation at $95^{\circ} \mathrm{C}$ for 5 minutes, followed by 30 cycles of $95^{\circ} \mathrm{C}$ for 1 minute, $60^{\circ} \mathrm{C}$ for 1 minute, and $72^{\circ} \mathrm{C}$ for 1 minute, with a final extension cycle of $72^{\circ} \mathrm{C}$ for 5 minutes. The automated Genetic Analyzer ABI 3130 XL (Applied Biosystems3130, Foster City, California, USA) was applied to directionally sequence PCR products using The Big Dye ${ }^{\circledR}$ Terminator v3.1 Cycle Sequencing Kit (Applied Biosystems3130, Foster City, California, USA).

\subsection{Computational analyses}

We used Bioinformatics predictive tools including Mutation Taster, Polyphen and SIFT (16) to assess possible effects of mutations on the protein structure. Databases including: Connexin deafness homepage (http://davinci.crg.es/deafness), the Single Nucleotide Polymorphism database (dbSNP) (http://www.ncbi.nlm. nih.gov/project/SNP) and the 1000 Genomes Project (http://browser.1000genomes.org) were investigated for novel variants.

\section{Results}

\subsection{Clinical Evaluation}

In the four-generation consanguineous family CHM-1, four individuals (III-3, IV-2, IV-2, IV-3), were affected by pre-lingual bilateral profound sensorineural HL (Figure 2A). The deaf siblings had normal parents and helped to correctly define the mode of inheritance as autosomal recessive. In accordance with the subjects' interviews indicating a pre-lingual onset of deafness,
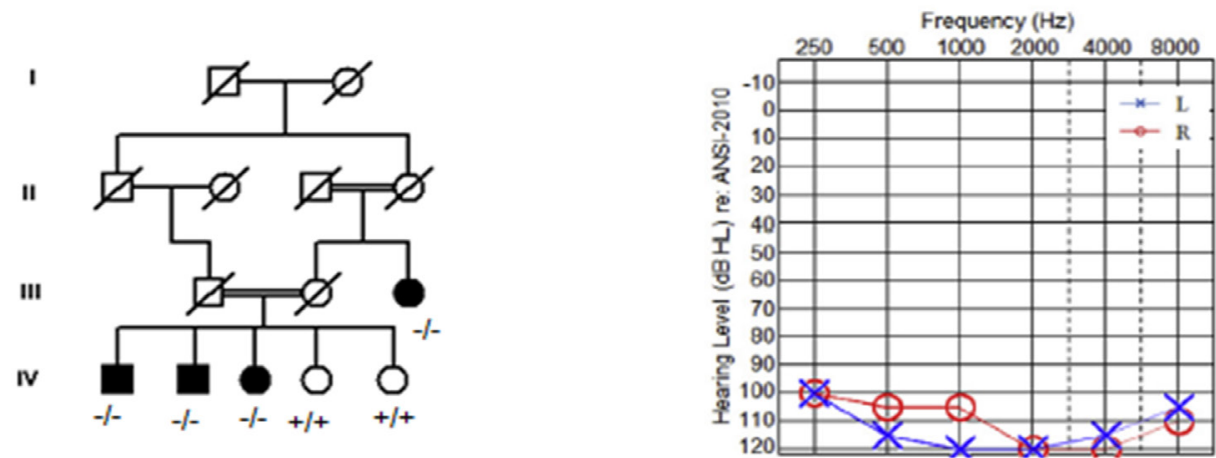

Figure 2. (A) Pedigree and segregation of the c.109_110insG variant in Iranian family CHM-1; - /-and +/+ respectively, show the homozygous (mutant) genotype and wild-type (WT) allele. (B) Right and left ear audiograms in the (IV: 2) patient. 

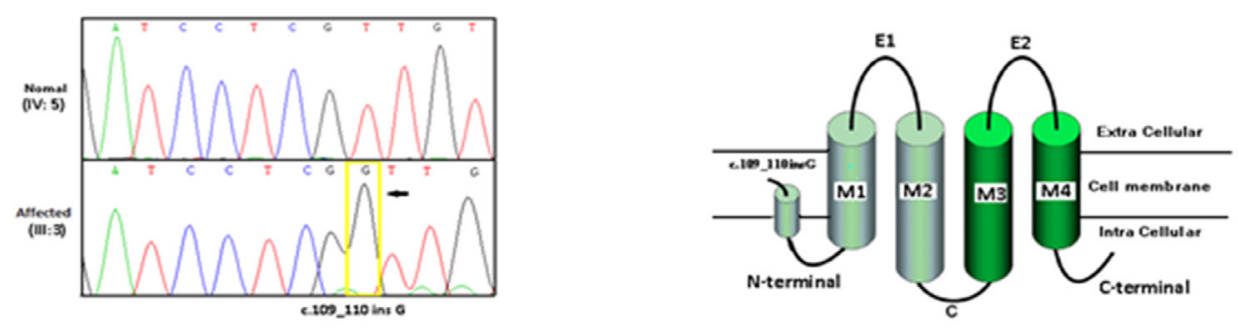

Figure 3. (A) Sequencing results of the c.109_110insG variant. (B) Schematic structure, domains and location of new variant of the Cx-26 protein in this study.

Table 1. Identified GJB2 variant and in silico analyses

\begin{tabular}{lllllllllll}
\hline Mutation & $\begin{array}{l}\text { Amino acid } \\
\text { change }\end{array}$ & $\begin{array}{l}\text { mutation } \\
\text { type }\end{array}$ & $\begin{array}{l}\text { Affected } \\
\text { domain }\end{array}$ & classification & \multicolumn{3}{c}{ Functional effect } & \multicolumn{2}{c}{$\begin{array}{l}\text { Segregates in } \\
\text { the family }\end{array}$} & ACMG \\
\hline c.109_110insG & Val37Gly fs*11 & Insertion & TM1 & Trancated & Damaging & 23 & Disease causing & Yes & Pathogenic \\
\hline
\end{tabular}

Normal (TV:5)

226 amino acids

Affected (III:3)

47 amino acid

Figure 4. The putative schematic representation of connexin26 protein and mutants in family CHM-1. The new variant c.109_110insG induced a frameshift mutation, caused a stop codon at position of amino acid 47, resulting in truncated connexin26 protein.

language acquisition was severely impaired in all affected individuals. More clinical examinations did not indicate any thyroid, skin, or ophthalmic abnormalities in affected members. Therefore, a syndromic form of HL was ruled out. The pedigree revealed several consanguineous marriages, suggesting ARNSHL in the offspring. Pure-tone audiometry in affected subjects showed flat audiograms characteristic of profound hearing impairment (Figure 2B).

\subsection{GJB2 Mutation Screening}

Sequencing of GJB2 showed a novel homozygous variant (c.109_110insG) in the three affected siblings and their deaf aunts (Figure 3A). This variant was not found in other normal hearing family members, and was unique to those affected. Additionally, no other mutation in GJB2 was identified in either of the subjects. c.109_110insG was not detected in 100 ethnicitymatched controls. All in silico programs predicted the c.109_110insG variant to have damaging effects (Table 1). Further analysis of c.109_110insG showed that the variant was not reported by 1000 genome project. This frameshift variant (p. Val37Gly*11) creates a stop codon at amino acids 47 , resulting in a truncated protein with only 47 residues (versus 226 residues in the intact protein) (Figure 4). This practically means complete deletion of about $75 \%$ of the Cx 26 protein. Figure 3 shows location of the variant in the first TM domain of the $\mathrm{Cx} 26$ protein.

\section{Discussion}

Various genes causing autosomal recessive nonsyndromic hearing loss (ARNSHL) has been identified from Iran; but mutations related to the GJB2 gene have been reported more frequently (17-19). Here, a novel homozygous variant identified by Sanger sequencing is reported within an Iranian family. The prevalence of $G J B 2$ mutations in Iran ranges from 4 to $38 \%$ in different ethnic groups (14). Such mutations are among the most frequent causes of HL in the world, and their prevalence exceeds over $50 \%$ in certain populations (20). GJB2, encodes the connexin 26 (Cx26) gap-junction channel protein that underlies both intercellular communication among supporting cells and homeostasis of the cochlear fluids, endolymph and perilymph. Connexin proteins that contain one cytoplasmic domain (CL), two extracellular domains (E 1-2), and four transmembrane (TM 1-4) domains are concentrated within the membrane as connexons or hemi channels. Newly-synthesized Cx26 monomers 


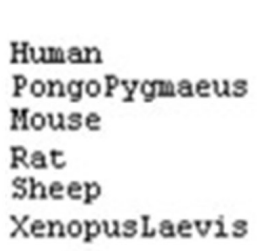

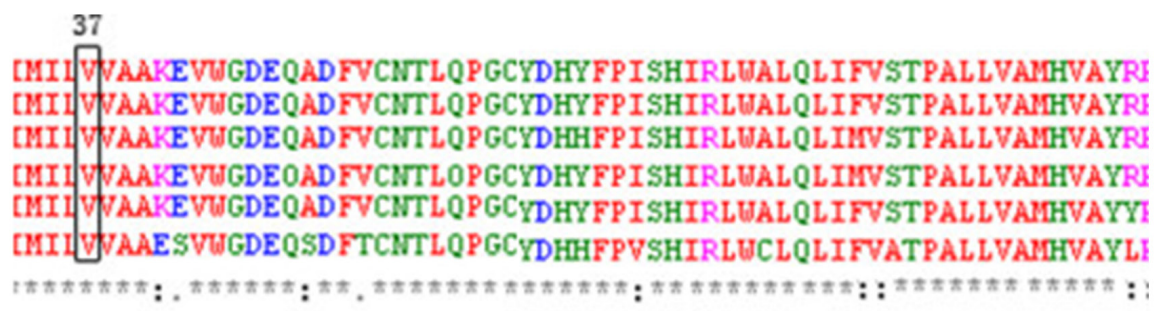

Figure 5. The identified variant c.109_110insG occurs at a highly conserved position (Val 37 ) in the Cx26 protein.

undergo conformational maturation and assembly into connexons when moving along the secretory pathway for delivery at the plasma membrane. Once there, $\mathrm{Cx} 26$ connexons may remain as so-called hemi channels, allowing for transport of diverse small molecules (less than $1 \mathrm{kDa}$ ) between the cytosol and the extracellular space. Until now, more than 100 mutations in GJB2 have been reported to cause ARNSHL. They involve a wide spectrum of missense, nonsense, frame shift and splice site mutations (http://davinci.crg.es/deafness). However, one truncated mutation (c.35delG) is most frequent in the majority of the Caucasian population, with the carrier frequency as high as $2-4 \%(21)$. Here, we identified a novel homozygous variant, $p$. Val37Gly*11 in the first TM domain of the Cx26 protein, which is highly conserved in all species studied, and among different connexions (Figure 5). This frameshift variant was predicted to result in a truncated protein missing parts of the TM1-4, intervening loops and C-terminal (Figure 3B) which hampers Cx26 folding and oligomerization, leading to retention at the endoplasmic reticulum (ER) and ultimately causing a total loss of function (22). Interestingly, ER retention of the truncated subunits may induce in some cases the unfolded protein response, which in turn may eventually lead to apoptosis of cells expressing those GJB2 mutant alleles (23). Unfortunately, there has been no in vitro functional assay to evaluate the mechanism by which GJB2 mutations cause HL. In this case, other available lines of evidence such as those derived from segregation studies should be incorporated to pinpoint true genetic causes. The ACMG guidelines advocated our finding (24). The frameshift variant is a null allele leading to a truncated protein (PVS1), the variant was seen in all affected family members and was not detected in normal subjects (PS4) and bioinformatics analyses support a deleterious effect on $\mathrm{Cx} 26$ protein (PP3). It is located in a validated domain (TM1), this variant reduces the length of the protein (PM4) and was not reported by the dbSNP database and 1000 genome project (PM2). Thus, the variant is categorized as being pathogenic.

This study should be a great supplement for the role of GJB2 mutations in the etiology of ARNSHL, in the Iranian population. Furthermore, our data support the view that the investigation of GJB2 mutations is still the primary step before moving on to next-generation sequencing (NGS) (25). The use of Sanger sequencing for detection of GJB2 mutations may be a faster and more economical diagnostic tool in individuals suffering with pre-lingual HL.

\section{Acknowledgements}

The author gratefully thanks all the patients who participated in this research.

\section{References}

1. Morton NE. Genetic epidemiology of hearing impairment. Ann N Y Acad Sci. 1991; 630:16-31.

2. Lucotte G, Mercier G. Meta-analysis of GJB2 mutation 35delG frequencies in Europe. Genet Test. 2001; 5:149152.

3. Kelsell DP, Dunlop J, Stevens HP, Lench NJ, Liang JN, Parry G, Mueller RF, Leigh IM. Connexin 26 mutations in hereditary non-syndromic sensorineural deafness. Nature. 1997; 387:80-83.

4. Azadegan-Dehkordi F, Ahmadi R, Koohiyan M, Hashemzadeh-Chaleshtori M. Update of spectrum c. $35 \mathrm{delG}$ and c. $-23+1 \mathrm{G}>\mathrm{A}$ mutations on the GJB2 gene in individuals with autosomal recessive nonsyndromic hearing loss. Ann Hum Genet. 2019; 83:1-10.

5. Koohiyan M, Azadegan-Dehkordi F, Koohian F, Hashemzadeh-Chaleshtori M. Genetics of hearing loss in north Iran population: An update of spectrum and frequency of GJB2 mutations. J Audiol Otol. 2019; 23:175-180.

6. Abe S, Usami S, Shinkawa H, Kelley PM, Kimberling WJ. Prevalent connexin 26 gene (GJB2) mutations in Japanese. J Med Genet. 2000; 37:41-43.

7. Lerer I, Sagi M, Malamud E, Levi H, Raas-Rothschild A, Abeliovich D. Contribution of connexin 26 mutations to nonsyndromic deafness in Ashkenazi patients and the variable phenotypic effect of the mutation 167delT. Am J Med Genet. 2000; 95:53-56.

8. Zarepour N, Koohiyan M, Taghipour-Sheshdeh A, Nemati-Zargaran F, Saki N, Mohammadi-Asl J, Tabatabaiefar MA, Hashemzadeh-Chaleshtori M. Identification and Clinical Implications of a Novel MYO15A Variant in a Consanguineous Iranian Family by Targeted Exome Sequencing. Audiol Neurootol. 2019; 24:25-31.

9. Koohiyan M. A systematic review of SLC26A4 mutations causing hearing loss in the Iranian population. Int J Pediatr Otorhinolaryngol. 2019; 125:1-5.

10. Koohiyan M, Reiisi S, Azadegan-Dehkordi F, Salehi M, 
Abtahi H, Hashemzadeh-Chaleshtori M, Noori-Daloii MR, Tabatabaiefar MA. Screening of 10 DFNB loci causing autosomal recessive non-syndromic hHearing loss in two Iranian pPopulations negative for GJB2 mutations. Iran J Public Health. 2019; 48:1704-1713.

11. Koohiyan M, Noori-Daloii MR, HashemzadehChaleshtori M, Salehi M, Abtahi H, Tabatabaiefar MA. A novel pathogenic variant in the CABP2 gene causes severe nonsyndromic hearing loss in a consanguineous Iranian family. Audiol Neurootol. 2019; 24:258-263.

12. Koohiyan M. Genetics of hereditary hearing loss in the Middle East: A systematic review of the carrier frequency of the GJB2 mutation (35delG). Audiol Neurootol. 2019; 24:161-165.

13. Koohiyan M, Azadegan-Dehkordi F, Koohian F, Abolhasani M, Hashemzadeh-Chaleshtori M. Genetics of hereditary hearing loss in east Iran population: A systematic review of GJB2 mutations. Intractable Rare Dis Res. 2019; 8:172-178.

14. Koohiyan M, Ahmadi A, Koohian F, Aghaei S, Amiri B, Hashemzadeh-Chaleshtori M. An update of spectrum and frequency of GJB2 mutations causing hearing loss in the south of Iran: A literature review. Int J Pediatr Otorhinolaryngol. 2019; 119:136-140.

15. Miller SA, Dykes DD, Polesky HF. A simple salting out procedure for extracting DNA from human nucleated cells. Nucleic Acids Res. 1988; 16:1215.

16. Kumar P, Henikoff S, Ng PC. Predicting the effects of coding non-synonymous variants on protein function using the SIFT algorithm. Nat Protoc. 2009; 4:1073-1081.

17. Azadegan-Dehkordi F, Bahrami T, Shirzad M, Karbasi G, Yazdanpanahi N, Farrokhi E, Koohiyan M, Tabatabaiefar MA, Hashemzadeh-Chaleshtori M. Mutations in GJB2 as major causes of autosomal recessive non-syndromic hearing loss: first report of c. 299-300delAT mutation in Kurdish population of Iran. J Audiol Otol. 2019; 23:2026.

18. Koohiyan M, Hashemzadeh-Chaleshtori M, Salehi M, Abtahi H, Reiisi S, Pourreza MR, Noori-Daloii MR, Tabatabaiefar MA. GJB2 mutations causing autosomal recessive non-syndromic hearing loss (ARNSHL) in two Iranian populations: Report of two novel variants. Int $\mathrm{J}$ Pediatr Otorhinolaryngol. 2018; 107:121-126.

19. Koohiyan M, Koohian F, Azadegan-Dehkordi F. GJB2- related hearing loss in central Iran: Review of the spectrum and frequency of gene mutations. Ann Hum Genet. 2019 Sep 11. doi: 10.1111/ahg.12354.

20. Gasparini P, Rabionet R, Barbujani G, Melçhionda S, Petersen M, Brøndum-Nielsen K, Metspalu A, Oitmaa E, Pisano M, Fortina P, Zelante L, Estivill X. High carrier frequency of the $35 \mathrm{delG}$ deafness mutation in European populations. Eur J Hum Genet. 2000; 8:19-23.

21. Green GE, Scott DA, McDonald JM, Woodworth GG, Sheffield VC, Smith RJ. Carrier rates in the midwestern United States for GJB2 mutations causing inherited deafness. JAMA. 1999; 281:2211-2216.

22. Xiao Z, Yang Z, Liu X, Xie D. Impaired membrane targeting and aberrant cellular localization of human Cx26 mutants associated with inherited recessive hearing loss. Acta Otolaryngol. 2011; 131:59-66.

23. Lindholm D, Korhonen L, Eriksson O, Kõks S. Recent insights into the role of unfolded protein response in ER stress in health and disease. Front Cell Dev Biol. 2017; $5: 48$.

24. Richards S, Aziz N, Bale S, Bick D, Das S, GastierFoster J, Grody WW, Hegde M, Lyon E, Spector E, Voelkerding K, Rehm H; ACMG Laboratory Quality Assurance Committee. Standards and guidelines for the interpretation of sequence variants: a joint consensus recommendation of the American College of Medical Genetics and Genomics and the Association for Molecular Pathology. Genet Med. 2015; 17:405-424.

25. Koohiyan M. Next generation sequencing and genetics of hereditary hearing loss in the iranian population: New insights from a systematic review. Int J Pediatr Otorhinolaryngol. 2019; 129:109756.

Received December 3, 2019; Revised January 5, 2020; Accepted January 17, 2020

*Address correspondence to:

Mahbobeh Koohiyan, Cancer Research Center, Shahrekord University of Medical Sciences, Shahrekord 8813833435, Iran.

E-mail:m_kouhiyan@yahoo.com

Released online in J-STAGE as advance publication February 3,2020 . 